\title{
Preface: To the Memory of Javier Chavarriga
}

\author{
Jaume Giné ${ }^{1}$ • Jaume Llibre ${ }^{2}$
}

Published online: 25 March 2016

(c) Springer International Publishing 2016

Javier Chavarriga obtained his degree in Mathematics at the Universitat de Barcelona in 1979 and finalized his Ph.D. in Mahtematics in 1985 at the same university. At the age of 30 he got an Associate Professor position at the Universitat de Barcelona and 15 years later he got the Full Professor position at the Universistat de Lleida. The mathematical activities of Javier included, among others, the supervision of four Ph.D. theses, five books, more than 60 research papers with more than 20 coauthors from different countries.

Javier's contributions to Dynamical systems started in the field of ordinary planar differential systems in the 90's, through a series of works on the center problem. His earliest publication is in 1994 following the work of his Ph.D. thesis. The integrability and the isochronicity problems have been other topics of his interest.

About the integrability problem of differential equations he has refined and generalized Darboux's classical results and its relations with the center problem.

He also has been interested in limit cycles, their existence, unicity, stability and location, and specially in the 16th Hilbert problem, using several methods of bifurcation theory.

He studied the isochronous center problem for polynomial differential systems of low degree. His main contribution in this subject was the paper: J. Chavarriga, M.

\footnotetext{
Jaume Giné

gine@matematica.udl.cat

Jaume Llibre

jllibre@mat.uab.cat

1 Departament de Matematica, Escola Politecnica Superior, Universitat de Lleida, Av. Jaume II, 69, 25001 Lleida, Spain

2 Departament de Matematiques, Universitat Autonoma de Barcelona, Edifici C, Bellaterra, 08193 Barcelona, Spain
} 
Sabatini, A survey of isochronous centers, Qual. Theory Dyn. Syst, 1 (1999), no. 1, $1-70$. Moreover this paper is one of the most cited paper in our journal QTDS and it becomes a classical reference in this area.

Javier Chavarriga was the first Editor-in-Chief together with Jaume Llibre of this journal.

This issue is dedicated to his memory after he died due to a hearth attack 10 years ago.

Jaume Giné and Jaume Llibre 